Research Article

\title{
Physical Manipulation of Calcium Oscillation in Shen-Larter Cell System
}

\author{
Hongkun Zuo \\ Department of Financial Science, Huainan Normal University, Huainan, Anhui 232038, China \\ Correspondence should be addressed to Hongkun Zuo; hk_zuo@163.com
}

Received 3 May 2015; Accepted 28 June 2015

Academic Editor: Dapeng P. Du

Copyright ( 2015 Hongkun Zuo. This is an open access article distributed under the Creative Commons Attribution License, which permits unrestricted use, distribution, and reproduction in any medium, provided the original work is properly cited.

Experimental observations show that calcium signals in response to external stimuli encode information via frequency modulation or alternatively via amplitude modulation. Here, by arguments of bifurcation theory and bifurcation control theorem, the amplitude and frequency manipulation of limit cycle in Shen-Larter cell system are investigated. A nonlinear feedback controller is then applied to the system, in order to assign the peak frequency to a prescribed value, such that bifurcation is minimized or eliminated in the closed-loop system. And numerical simulations are presented in order to illustrate the theoretical analysis.

\section{Introduction}

Biological cells draw on intracellular and extracellular calcium sources to generate signals that transduce stimulation into physiological output. Changes in cellular calcium concentration control a wide range of physiological processes $[1,2]$. The complex behavior can be understood through bifurcation analysis. A bifurcation is a qualitative change in the number of candidate operating conditions of a nonlinear system when the parameters are quasistatically varied [3]. One important attribute of a bifurcation is the direction, or stability, of the bifurcation. It is known that bifurcation properties of nonlinear system can be modified via various feedback control method, thereby achieving some desirable dynamical behaviors.

In general, bifurcation control deals with using a control input to modify the characteristics of a parameterized nonlinear system. The control can be static or dynamic feedback control or open loop control law. The bifurcation control objective can be delaying the onset of an inherent bifurcation, optimization of performance index near bifurcation, stabilization or reduction of the amplitude of some limit cycle emerging from bifurcation, changing the parameter value of an existing bifurcation point, reshaping of the bifurcation diagram, or a combination of these [4-6]. The work has been applied to control problems in engineering, biological, and physicochemical systems.
Representative approaches employing linear or nonlinear state-feedback controls were proposed by Abed and Fu in [7]. In [4], Wang and Abed apply a washout filter-aided dynamic feedback controller to bifurcation control. Harmonic balance approximations method was used to design the controller by Berns et al. Kang [8] investigated the bifurcation control with quadratic invariants in normal forms. In this paper, we focus on the analysis and control of the amplitudes of limit cycle in Shen-Larter cell system, and the control law is achieved by computing the stability index (or curvature coefficient).

The organization of the paper is as follows. Section 2 introduces the nonlinear system under study and shows all equilibrium points [9]. In Section 3, we use bifurcation control theory [10-12] to analyze the amplitude of limit cycle in the original system. Section 4 gives the amplitude control for limit cycle in Shen-Larter cell system. Some numerical simulations are presented for illustrating the theory in Section 5. Finally, our conclusions are presented in Section 6.

\section{Model}

The Shen-Larter model is considered in this paper as a typical example of calcium oscillation in nonexcitable cells [13]. The mechanisms of this model are based on the calcium-induced calcium release (CICR) as well as the inositol trisphosphate cross coupling (ICC), which contains three variables, namely, 
the concentrations of free calcium in the cytosol $(x)$ and in the internal pool $(y)$ and $\mathrm{IP}_{3}$ concentration $(z)$ :

$$
\begin{aligned}
& \frac{d x}{d t}=J_{\text {ch }}+J_{\text {leak }}-J_{\text {pump }}+J_{\text {in }}-J_{\text {out }} \\
& \frac{d y}{d t}=J_{\text {pump }}-J_{\text {ch }}-J_{\text {leak }} \\
& \frac{d z}{d t}=J_{+}-J_{-}
\end{aligned}
$$

where

$$
\begin{aligned}
J_{\text {ch }} & =k_{\text {ch }}\left(\frac{z^{4}}{z^{4}+K_{1}^{4}}\right)\left(\frac{K_{4} x}{\left(x+K_{4}\right)\left(x+K_{5}\right)}\right)^{3} y, \\
J_{\text {leak }} & =k_{\text {leak }} y \\
J_{\text {pump }} & =k_{\text {pump }} \frac{x^{2}}{x^{2}+K_{2}^{2}}, \\
J_{\text {in } 1} & =k_{\text {in } 1} r+k_{\text {in } 2} \\
J_{\text {out }} & =k_{\text {out }} x, \\
J_{+} & =k_{+} r \frac{x}{x+K_{3}}, \\
J_{-} & =k_{-} z .
\end{aligned}
$$

Parameters are made as follows if not otherwise stated: $k_{\mathrm{ch}}=3000 \mu \mathrm{Ms}^{-1}, k_{\text {leak }}=1.0 \mathrm{~s}^{-1}, k_{\text {pump }}=50 \mu \mathrm{Ms}^{-1}, k_{\text {in } 1}=$ $4 \mu \mathrm{Ms}^{-1}, k_{\text {in2 }}=1 \mu \mathrm{Ms}^{-1}, k_{\text {out }}=10 \mathrm{~s}^{-1}, k_{+}=4.0 \mu \mathrm{Ms}^{-1}, k_{-}=$ $2 \mu \mathrm{Ms}^{-1}, K_{1}=K_{2}=0.2 \mu \mathrm{Ms}^{-1}, K_{3}=1 \mu \mathrm{M}$, and $K_{4}=K_{5}=$ $0.69 \mu \mathrm{M}$; we define $r$ as bifurcation parameter.

When $r=0.2345$, the equilibrium points of system are as follows: $o_{1}=(0.1938,18.4885,0.0761)$ and $o_{1}$ is unstable; when $r=0.6859$, the equilibrium points of system are as follows: $o_{2}$ $=(0.3744,1.1486,0.3737)$ and $o_{2}$ is unstable. By applying Hopf bifurcation theorem, we obtain subcritical Hopf bifurcation of equilibria which are demonstrated in Figure 1.

\section{Amplitude of Limit Cycle in the Original System}

We consider the equilibrium point $o_{1}$ for $r=0.2345$ and calculate the eigenvalues of system (1) at $(0.1938,18.4885$, $0.0761)$ that are $\lambda_{1,2}(r)=\alpha(r) \pm i \omega(r), \lambda_{3}(r)$. It is known that

$$
\alpha^{\prime}(0.2345)=\left.\frac{\partial \alpha(r)}{\partial r}\right|_{r=0.2345}=0.4160
$$

Suppose that

$$
\begin{aligned}
\left(\begin{array}{l}
x \\
y \\
z
\end{array}\right)= & \left(\begin{array}{c}
0.1938 \\
18.4885 \\
0.0761
\end{array}\right)+P\left(\begin{array}{l}
u \\
v \\
w
\end{array}\right), \\
\text { where } P & =\left(\begin{array}{ccc}
0 & 1 & 1 \\
18.7853 & -1.0387 & -0.8918 \\
-0.0817 & 0.3072 & -0.0073
\end{array}\right) .
\end{aligned}
$$

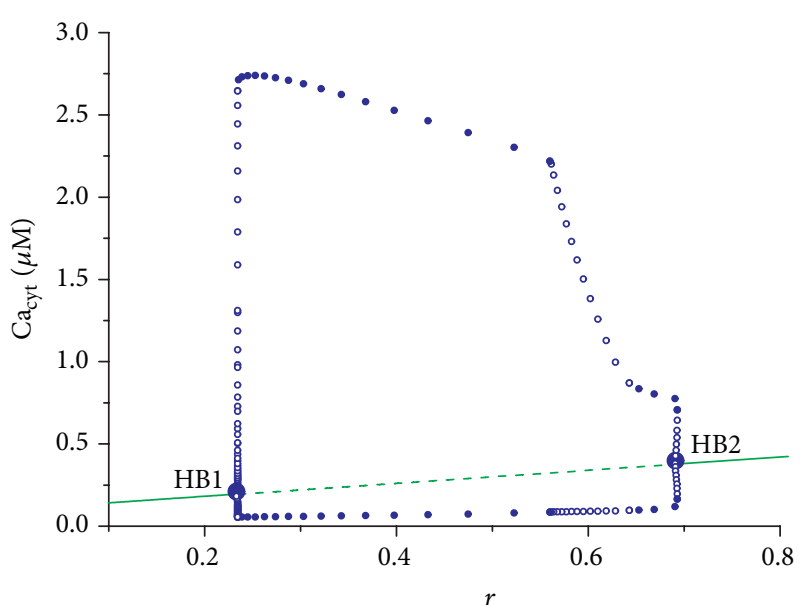

FIgURE 1: The equilibrium curve of $\left(r, \mathrm{Ca}_{\text {cyt }}\right)$ for system (1).

Substituting (4) into system (1), we obtain

$$
\begin{aligned}
\left(\begin{array}{c}
\dot{u} \\
\dot{v} \\
\dot{w}
\end{array}\right)= & \left(\begin{array}{ccc}
0 & -0.5323 & 0 \\
0.5323 & 0 & 0 \\
0 & 0 & -92.4310
\end{array}\right) \cdot\left(\begin{array}{l}
u \\
v \\
w
\end{array}\right) \\
& +\left(\begin{array}{l}
Q_{1} \\
Q_{2} \\
Q_{3}
\end{array}\right),
\end{aligned}
$$

where

$$
\begin{aligned}
Q_{1}= & -0.09925049 u+0.0471429 v-0.47273156 w \\
& +0.0233562 a+0.275 b-5.4203985 c \\
& -0.10547653 \\
Q_{2}= & 0.39594975 u-2.33607963 v-0.328835 w \\
& +2.9885618 a-1.085 b+21.3859359 c \\
& -0.08372397, \\
Q_{3}= & 17.85705025 u-8.70262037 v+81.868035 w \\
& -2.9885618 a-48.915 b+964.141064 c \\
& +18.57222397, \\
a= & \frac{\sigma_{2}}{\sigma_{2}+1}, \\
b= & \frac{\sigma_{2}^{2}}{\sigma_{2}^{2}+0.04}, \\
c= & \frac{\sigma_{1} \sigma_{2}^{3} \sigma_{3}}{\left(\sigma_{1}+0.0016\right) \sigma_{4}},
\end{aligned}
$$




$$
\begin{aligned}
& \sigma_{1}=(0.0817 u-0.3072 v+0.0073 w-0.0761)^{4}, \\
& \sigma_{2}=v+w+0.1938, \\
& \sigma_{3}=18.7853 u-1.0387 v-0.8918 w+18.4885, \\
& \sigma_{4}=(v+w+0.8838)^{6} .
\end{aligned}
$$

In order to facilitate discussion, we make the following hypothesis:

$$
\begin{aligned}
\left(t_{1}, t_{2}, t_{3}\right) & =(u, v, w), \\
L_{j k}^{i} & =\left.\frac{\partial Q_{i}}{\partial t_{j} \partial t_{k}}\right|_{\left(t_{1}, t_{2}, t_{3}, r\right)=(0,0,0,0.2345)}, \\
L_{j k s}^{i} & =\left.\frac{\partial Q_{i}}{\partial t_{j} \partial t_{k} \partial t_{s}}\right|_{\left(t_{1}, t_{2}, t_{3}, r\right)=(0,0,0,0.2345)} .
\end{aligned}
$$

We calculate the following characteristic quantities for $r=$ 0.2345 and $(u, v, w)=(0,0,0)$

$$
\begin{aligned}
& g_{20}=0.25\left(L_{11}^{1}-L_{22}^{1}+2 L_{12}^{2}+i\left(L_{11}^{2}-L_{22}^{2}-2 L_{12}^{1}\right)\right), \\
& g_{11}=0.25\left(L_{11}^{1}+L_{22}^{1}+i\left(L_{11}^{2}+L_{22}^{2}\right)\right), \\
& G_{110}=0.5\left(L_{13}^{1}+L_{23}^{2}+i\left(L_{13}^{2}-L_{23}^{1}\right)\right), \\
& G_{101}=0.5\left(L_{13}^{1}-L_{23}^{1}+i\left(L_{13}^{2}+L_{23}^{1}\right)\right), \\
& w_{11}=\frac{1}{4 \times 92.43}\left(L_{11}^{3}+L_{22}^{3}\right), \\
& w_{20}=\frac{1}{4 \times(2 \times 0.5323 i+92.431)} \cdot\left(L_{11}^{3}-L_{22}^{3}\right. \\
& \left.\quad-2 i L_{12}^{3}\right), \\
& G_{21}=\frac{1}{8}\left(L_{111}^{1}+L_{122}^{1}+L_{112}^{2}+L_{222}^{2}\right. \\
& \left.\quad+i\left(L_{111}^{2}+L_{122}^{2}-L_{112}^{1}-L_{222}^{1}\right)\right)
\end{aligned}
$$

and get that

$$
\begin{aligned}
g_{20} & =0.7240-12.8285 i, \\
g_{11} & =-4.4838+16.7853 i, \\
G_{110} & =13.5814+2.1357 i, \\
G_{101} & =-12.7404-5.5436 i, \\
w_{11} & =8.6249, \\
w_{20} & =-8.4743+1.8988 i, \\
G_{21} & =91.9212+11.9489 i .
\end{aligned}
$$
by

Then the curvature coefficient of limit cycle is described

$$
\begin{aligned}
\sigma & =\operatorname{Re}\left\{\frac{g_{20} g_{11}}{2 \times 0.5323} i+G_{110} w_{11}+\frac{G_{21}+G_{101} w_{20}}{2}\right\} \\
& =156.8140339 .
\end{aligned}
$$

For $|r-0.2345| \ll 1$, the amplitude of limit cycle of system (1) is

$$
\begin{aligned}
R & \ll \sqrt{-\frac{\alpha^{\prime}(0.2345)}{\sigma}(r-0.2345)} \\
& =0.0515 \sqrt{r-0.2345} .
\end{aligned}
$$

\section{Amplitude Control}

For the purpose of not changing the equilibrium and bifurcation parameter of system (1), we assume that the nonlinear feedback controller is $U(X, r)=\left[\begin{array}{lll}k(y-18.4885)^{2} & 0 & 0\end{array}\right]^{T}$, and then the model (1) can be rewritten as

$$
\begin{aligned}
& \frac{d x}{d t}=J_{\text {ch }}+J_{\text {leak }}-J_{\text {pump }}+J_{\text {in }}-J_{\text {out }} \\
& \quad+k(y-18.4885)^{2}, \\
& \frac{d y}{d t}=J_{\text {pump }}-J_{\text {ch }}-J_{\text {leak }}, \\
& \frac{d z}{d t}=J_{+}-J_{-} .
\end{aligned}
$$

According to the analysis of AM of limit cycle, we have

$$
\begin{aligned}
\left(\begin{array}{c}
\dot{u} \\
\dot{v} \\
\dot{w}
\end{array}\right)= & \left(\begin{array}{ccc}
0 & -0.5323 & 0 \\
0.5323 & 0 & 0 \\
0 & 0 & -92.4310
\end{array}\right)\left(\begin{array}{l}
u \\
v \\
w
\end{array}\right) \\
& +\left(\begin{array}{c}
\widetilde{Q}_{1} \\
\widetilde{Q}_{2} \\
\widetilde{Q}_{3}
\end{array}\right),
\end{aligned}
$$

where

$$
\begin{aligned}
& \left(\begin{array}{c}
\widetilde{Q}_{1} \\
\widetilde{Q}_{2} \\
\widetilde{Q}_{3}
\end{array}\right)=\left(\begin{array}{l}
Q_{1} \\
Q_{2} \\
Q_{3}
\end{array}\right)+\left(\begin{array}{c}
U_{1} \\
U_{2} \\
U_{3}
\end{array}\right), \\
& \left(\begin{array}{c}
U_{1} \\
U_{2} \\
U_{3}
\end{array}\right)=\left(\begin{array}{lll}
0.0478 & 0.0533 & 0.0249 \\
0.0356 & 0.0139 & 3.1861 \\
0.9644 & -0.0139 & -3.1861
\end{array}\right) U(X, r) .
\end{aligned}
$$


Similar to previous analysis, we calculate that

$$
\begin{aligned}
\widetilde{g}_{20} & =g_{20}+k(7.7136+6.9568 i), \\
\widetilde{g}_{11} & =g_{11}+k(8.4598+6.3006 i), \\
\widetilde{G}_{110} & =G_{110}+k(-0.7678-0.6407 i), \\
\widetilde{G}_{101} & =G_{101}+k(-0.8338-0.5521 i), \\
\widetilde{w}_{11} & =w_{11}+1.8466 k, \\
\widetilde{w}_{20} & =w_{20}+k(1.8374+0.1824 i), \\
\widetilde{G}_{21} & =G_{21} .
\end{aligned}
$$

Then we have the curvature coefficient of limit cycle:

$$
\begin{aligned}
\widetilde{\sigma}= & \operatorname{Re}\left\{\frac{\widetilde{g}_{20} \widetilde{g}_{11}}{2 \times 0.5323} i+\widetilde{G}_{110} \widetilde{w}_{11}+\frac{\widetilde{G}_{21}+\widetilde{G}_{101} \widetilde{w}_{20}}{2}\right\} \\
& =\sigma+\operatorname{Re}\left\{k^{2}(-103.0668+18.3568 i)\right. \\
& +k(16.6443-66.7055 i)\}=156.8140 \\
& -103.0668 k^{2}+16.6443 k .
\end{aligned}
$$
(12) is

For $|r-0.2345| \ll 1$, the amplitude of limit cycle of system

$$
\widetilde{R} \ll \sqrt{-\frac{\alpha^{\prime}(0.2345)}{\tilde{\sigma}}(r-0.2345) .}
$$

Furthermore, define $P=R / \widetilde{R}$; then

$$
P=\sqrt{1+0.1061 k-0.6573 k^{2}} \text {. }
$$

With these results that are in good agreement with experimental findings, the correct control parameter in the range $-1.1554<k<1.3169$ can be accomplished if the appropriate conditions are fulfilled [14]. The goal is to indirectly vary the value of $P$, thereby controlling the amplitudes of the limit cycles. The results are depicted in Figure 5.

\section{Numerical Simulation}

Considering the original system (1), Figure 2 indicates time series for $r=0.2345$ and Figure 3 shows the phase diagram in $\left(\mathrm{Ca}_{\text {cyt }}, \mathrm{Ca}_{\mathrm{er}}\right)$-space of system (1) for $r=0.2345$. System (1) has a nonhyperbolic equilibrium. It is concluded that system (1) has subcritical Hopf bifurcation at $o_{1}=(0.1938,18.4885$, 0.0761 ) for $r=0.2345$.

As expected the amplitude and frequency of system (1) are changed by the nonlinear feedback controller. Otherwise, the equilibrium and the location of the Hopf bifurcation points remain the same, which can be seen from Figure 4 . The green and blue lines of the curves represent the time series with and without control when varying the parameter $k$.

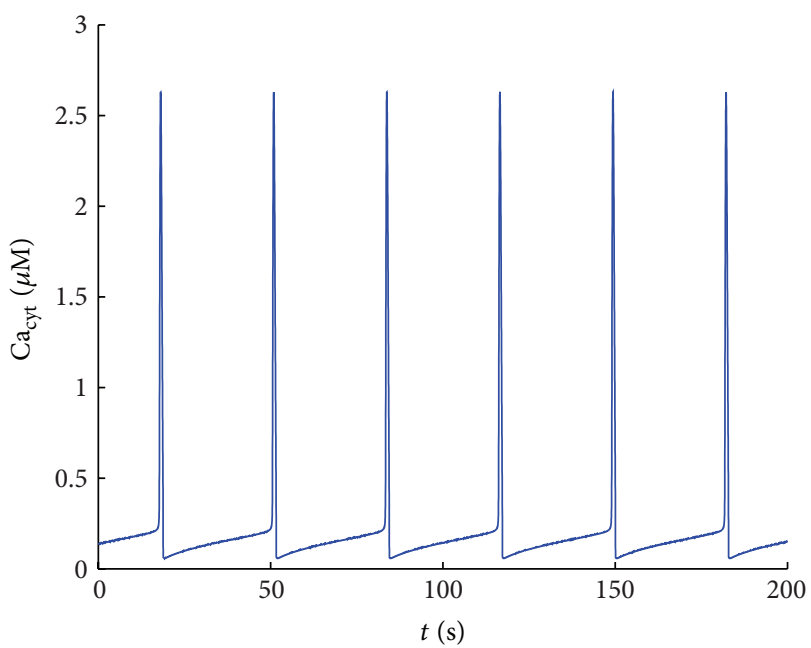

Figure 2: Time course of $\mathrm{Ca}_{\text {cyt }}$ for $r=0.2345$.

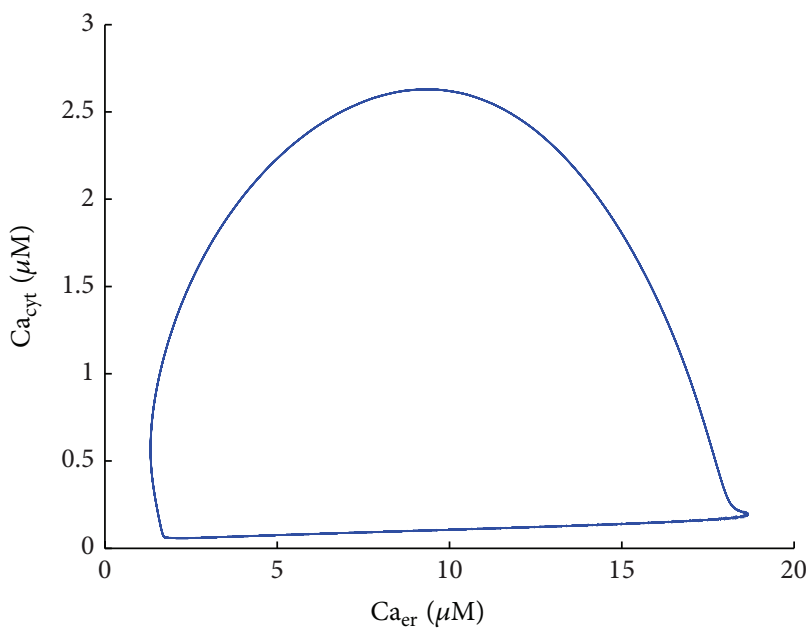

FIGURE 3: Trajectory in phase space for $r=0.2345$.

It is concluded that

(1) for $k=-0.004$ and $k=-0.053$, there are six peaks in blue line graphics comparing to those four peaks and three peaks in green line curve, as shown in Figures 4(a) and 4(b); its effect is to decrease the frequency by the feedback law $U(x, r)$;

(2) for $k=0.0066$ and $k=0.01644$, there are six peaks in blue line graphics comparing to those seven peaks and eight peaks in green line curve, as shown in Figures $4(\mathrm{c})$ and $4(\mathrm{~d})$; its effect is to increase the frequency by the feedback law $U(x, r)$.

It shows that an increase in frequency was observed for the calcium oscillations, when the concentration of agonist is raised high enough. This phenomenon can also qualitatively reproduce experimental data with appropriate values for parameter [15].

For the sake of comparing the variation of the amplitude of the limit cycles when $r=0.2345$, in Figure 5, we include 


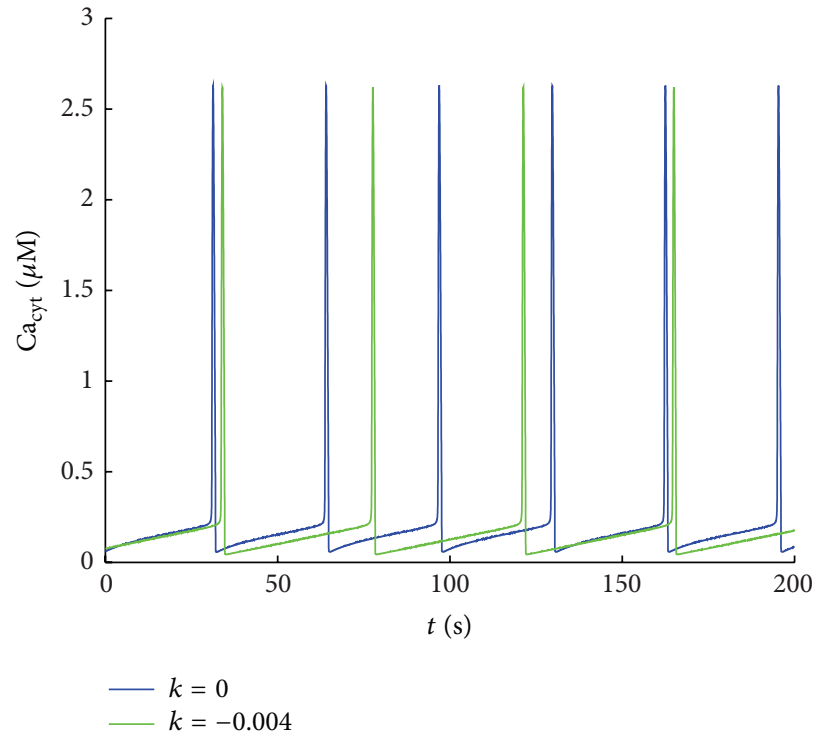

(a) $k=-0.004$

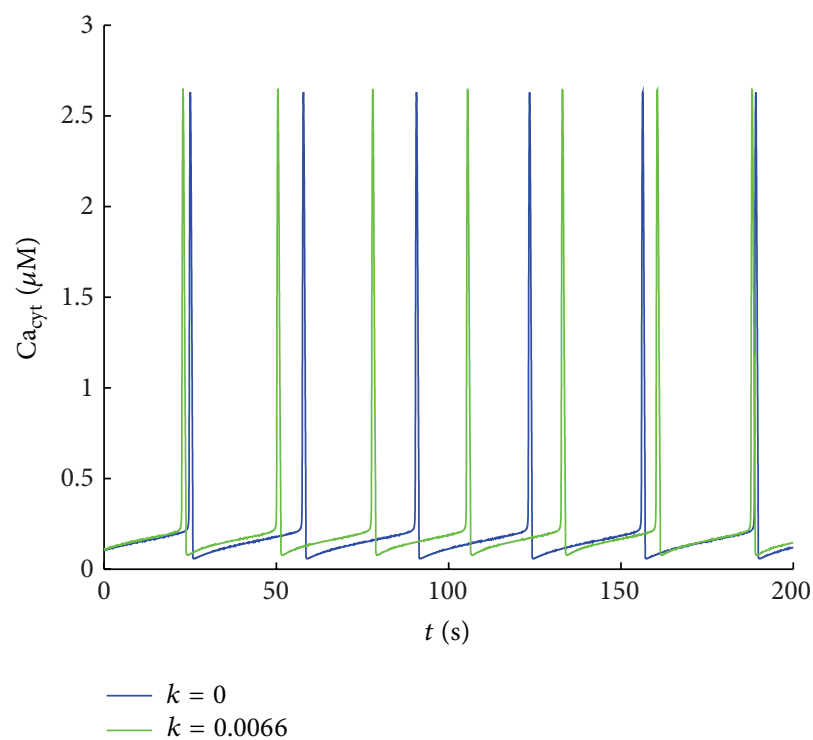

(c) $k=0.0066$

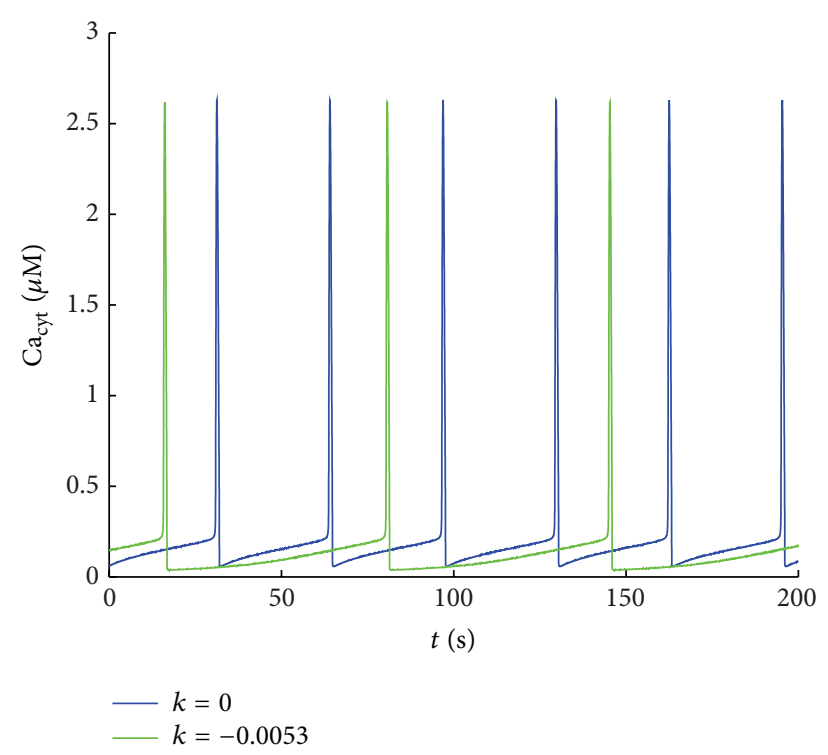

(b) $k=-0.0053$

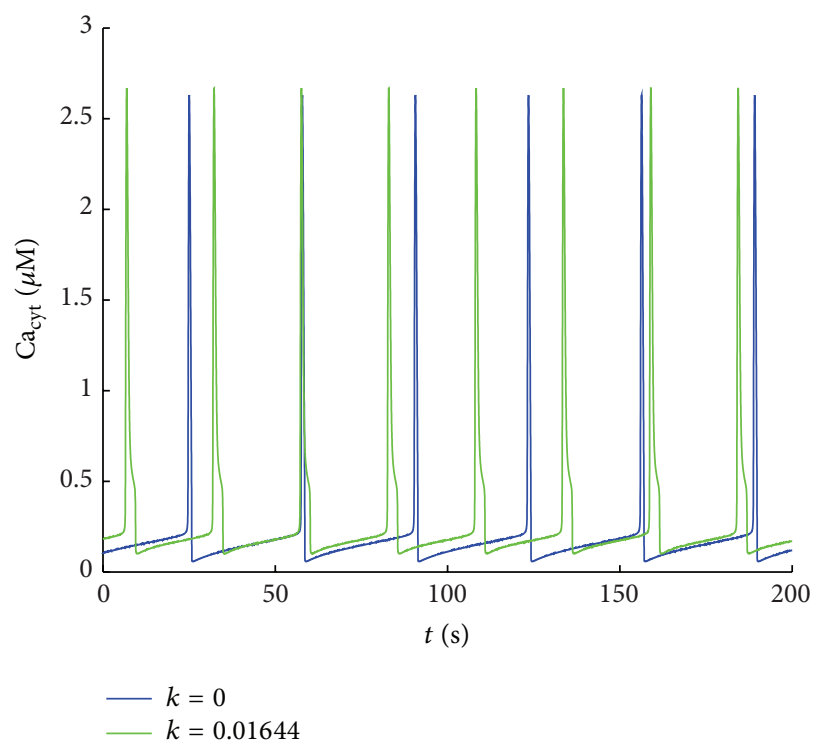

(d) $k=0.01644$

FIGURE 4: Time course of $\mathrm{Ca}_{\text {cyt }}$ for $r=0.2345$ under feedback law $U(x, r)$.

the original (without control) oscillatory solution and the one obtained by using the varying controller parameter $k$. It represents that the limit cycle under feedback law $U(x, r)$ and the predicted solutions are both difficult to be distinguished from the limit cycle for the value of $r=0.2345$. Figure 5 shows that the amplitude of the limit cycles can be controlled by varying the parameter $k$. In this case, we have reproduced the temporal sequence similar to those recorded in experiments [16].

\section{Conclusion}

We investigate the amplitude manipulation of Shen-Larter calcium oscillation model. A nonlinear feedback controller is designed for the amplitude control of the limit cycle. To ensure the effective control, the formula for amplitude of limit cycle is obtained by calculating the coefficient of curvature, and then the formula is used for the determination of the appropriate values of the control parameter. It is shown that the appropriate values of control parameter can be found through the formula. Simulations confirm our theoretical analysis results. Future studies should be undertaken to study amplitude and frequency control of calcium oscillation with time delay in detail.

Our numerical simulations of intracellular free calcium oscillation approximately reproduce the range of agonistinduced calcium responses via at least two modalities. It shows good agreement with experiments in that these 


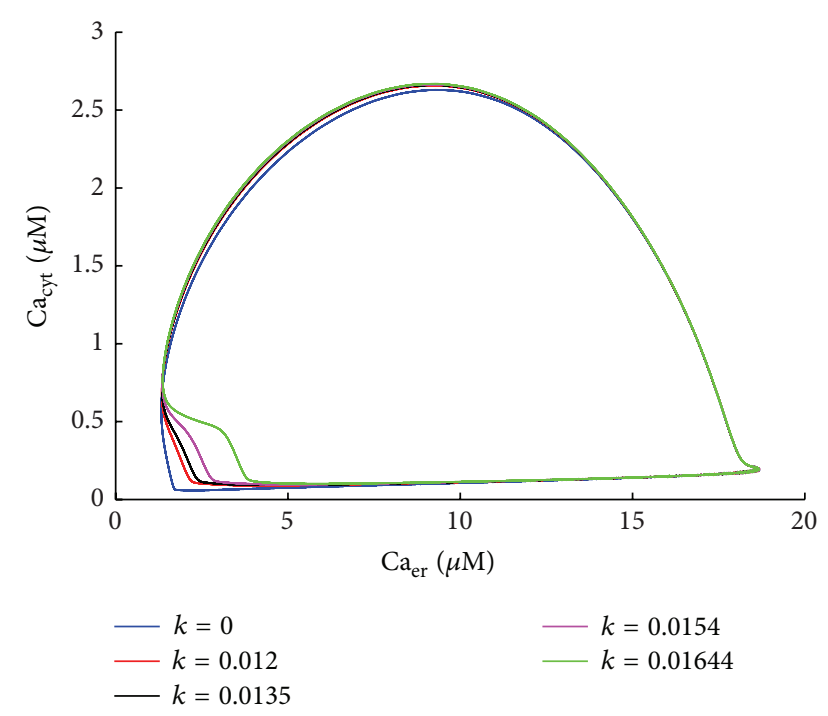

Figure 5: Trajectory in phase space for $r=0.2345$ under control.

calcium oscillations could persist for as long as receptors are activated at the cell surface. Comparing with experimental observations, we hope that the results may provide a guide for interpreting the previous experimental observations in other cell systems, where different types of calcium channels are possibly thought to be involved in calcium signaling.

\section{Conflict of Interests}

The author declares that there is no conflict of interests regarding the publication of this paper.

\section{Acknowledgments}

This work is supported by the National Natural Science Foundation of China (nos. 11202083 and 11372017) and the research foundation of Huainan Normal University (2014xj11zd and 2014xk07zd).

\section{References}

[1] M. Falcke, "Reading the patterns in living cells-the physics of $\mathrm{Ca}^{2+}$ signaling," Advances in Physics, vol. 53, no. 3, pp. 255-440, 2004.

[2] C.-L. Zhu, Y. Jia, Q. Liu, L.-J. Yang, and X. Zhan, "A mesoscopic stochastic mechanism of cytosolic calcium oscillations," Biophysical Chemistry, vol. 125, no. 1, pp. 201-212, 2007.

[3] V. I. Arnold, Geometrical Methods in the Theory of Ordinary Differential Equations, Springer, New York, NY, USA, 1988.

[4] H. O. Wang and E. H. Abed, "Bifurcation control of a chaotic system," Automatica, vol. 31, no. 9, pp. 1213-1226, 1995.

[5] A. Tesi, E. H. Abed, R. Genesio, and H. O. Wang, "Harmonic balance analysis of period-doubling bifurcations with implications for control of nonlinear dynamics," Automatica, vol. 32, no. 9, pp. 1255-1271, 1996.

[6] H. O. Wang and E. H. Abed, "Control of nonlinear phenomena at the inception of voltage collapse," in Proceedings of the
American Control Conference, pp. 2017-2075, San Francisco, Calif, USA, 1993.

[7] E. H. Abed and J. H. Fu, "Local feedback stabilization and bifurcation control, I. Hopf bifurcation," Systems \& Control Letters, vol. 7, no. 1, pp. 11-17, 1986.

[8] W. Kang, "Bifurcation and normal form of nonlinear control systems. Part I," SIAM Journal on Control and Optimization, vol. 36, no. 1, pp. 193-212, 1998.

[9] H. Zuo, Q. Ji, and Y. Zhou, "Hopf bifurcation and numerical simulation in a calcium oscillation model," Applied Mechanics and Materials, vol. 226-228, pp. 510-515, 2012.

[10] G. Chen, J. L. Moiola, and H. O. Wang, "Bifurcation control: theories, methods, and applications," International Journal of Bifurcation and Chaos in Applied Sciences and Engineering, vol. 10, no. 3, pp. 511-548, 2000.

[11] S. Zhou, X. Lin, and H. Li, "Chaotic synchronization of a fractional-order system based on washout filter control," Communications in Nonlinear Science and Numerical Simulation, vol. 16, no. 3, pp. 1533-1540, 2011.

[12] X. Wang, W. Zhang, and W. Chen, "Amplitude control and Hopf bifurcation anti-control of the Newton-Leipnik system," The International Journal of Nonlinear Science, vol. 13, no. 1, pp. 5863, 2012.

[13] M. Perc and M. Marhl, "Different types of bursting calcium oscillations in non-excitable cells," Chaos, Solitons and Fractals, vol. 18, no. 4, pp. 759-773, 2003.

[14] D. D. Friel, " $\mathrm{Ca}^{2+}$ oscillations in sympathetic neurons: an experimental test of a theoretical model," Biophysical Journal, vol. 68, no. 5, pp. 1752-1766, 1995.

[15] Y.-X. Li, J. Rinzel, J. Keizer, and S. S. Stojilković, "Calcium oscillations in pituitary gonadotrophs: comparison of experiment and theory," Proceedings of the National Academy of Sciences of the United States of America, vol. 91, no. 1, pp. 58-62, 1994.

[16] A. A. Borges, D. Salter, S. Kadar, and S. B. Symington, The development of a comprehensive mechanism for intracellular calcium oscillations: $a$ theoretical approach and an experimental validation [Pell Scholars and Senior Theses], Department of Chemistry Salve Regina University, 2010. 


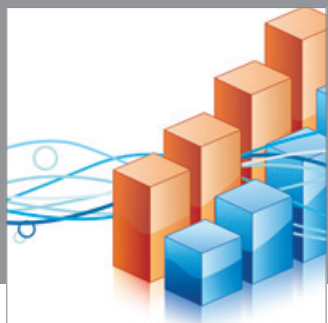

Advances in

Operations Research

mansans

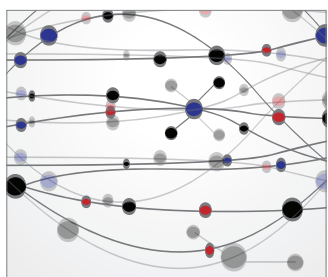

The Scientific World Journal
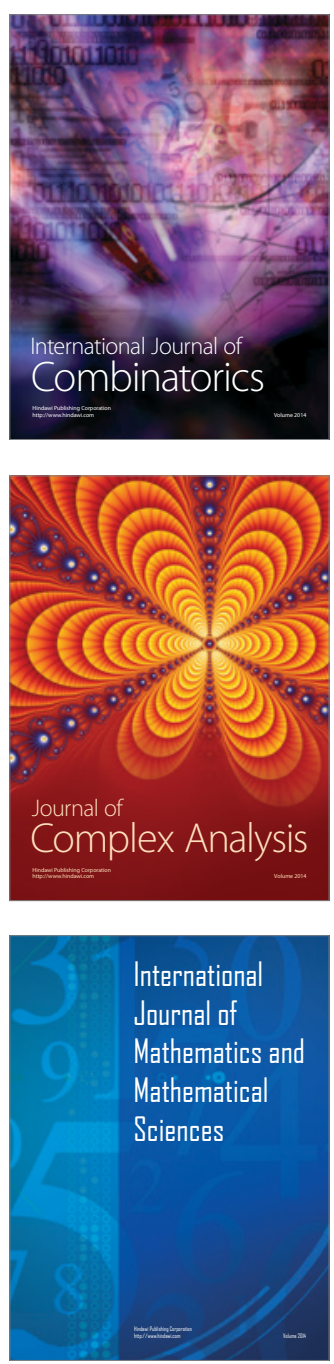
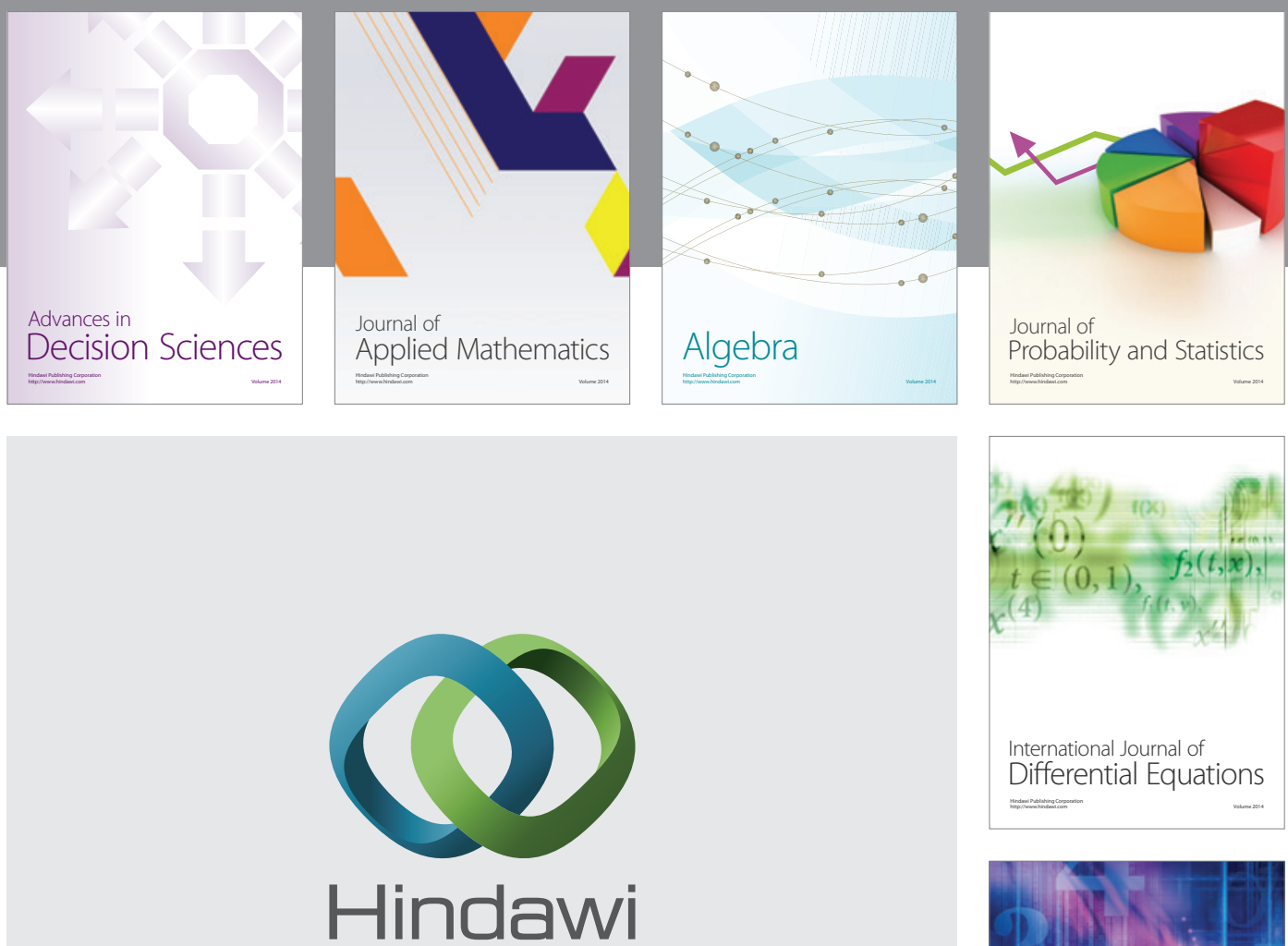

Submit your manuscripts at http://www.hindawi.com
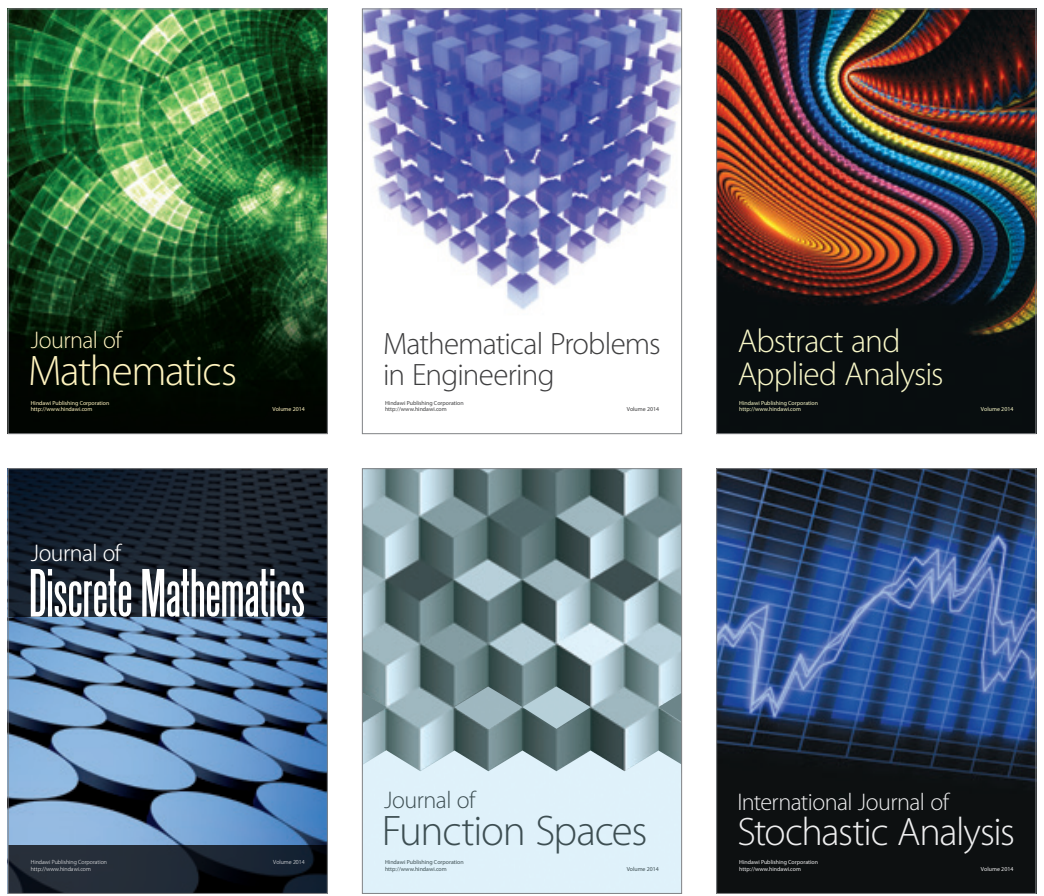

Journal of

Function Spaces

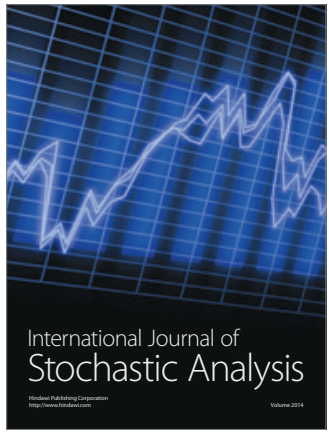

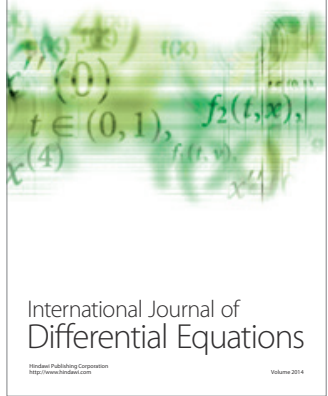
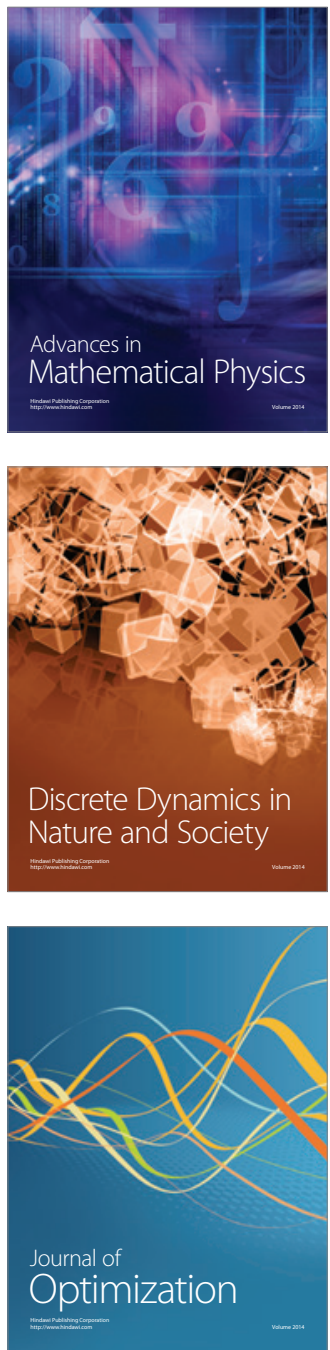